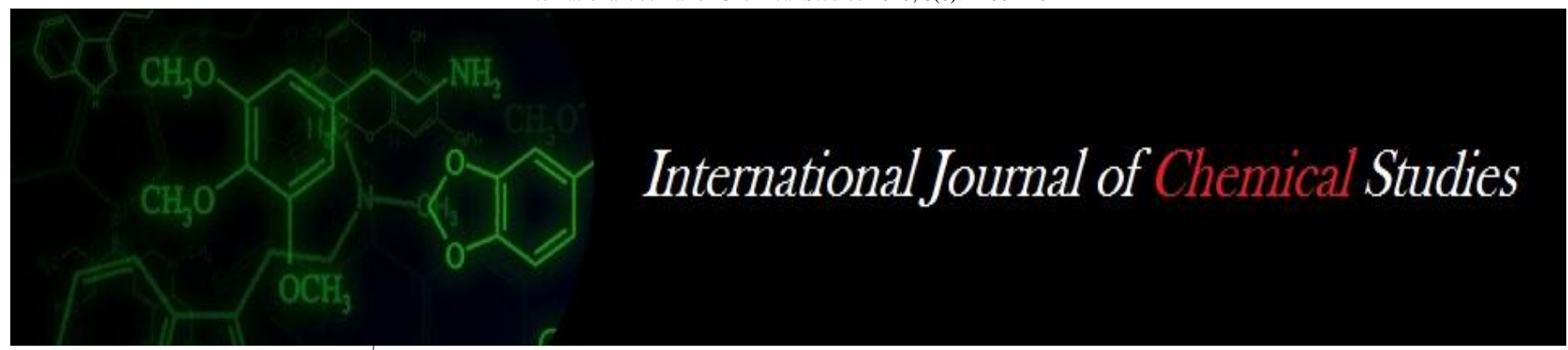

P-ISSN: 2349-8528

E-ISSN: 2321-4902

www.chemijournal.com

IJCS 2020; 8(6): 1100-1102

(C) 2020 IJCS

Received: 26-08-2020

Accepted: 13-10-2020

\section{Karde RY}

College of Agriculture,

Vasantrao Naik Marathwada

Krishi Vidyapeeth, Latur,

Maharashtra, India

\section{Chavan PG}

Assistant Professor,

College of Agriculture,

Usmanabad, Maharashtra, India

\section{Wakchaure BM}

College of Agriculture,

Vasantrao Naik Marathwada

Krishi Vidyapeeth, Latur,

Maharashtra, India
Corresponding Author:

\section{Karde RY}

College of Agriculture,

Vasantrao Naik Marathwada

Krishi Vidyapeeth, Latur,

Maharashtra, India

\section{Chemical weed management in maize using pre and post emergence herbicides}

\author{
Karde RY, Chavan PG and Wakchaure BM
}

DOI: https://doi.org/10.22271/chemi.2020.v8.i6p.10908

\begin{abstract}
A field experiment was conducted during kharif 2018-19 College of Agriculture, Latur to find out the effective herbicides for control of weeds in maize (Zea mays L.). The experiment comprises of ten treatment combinations and were compared with weed free and weedy check and laid out in randomized block design and replicated three times. Application of pendimethalin $1000 \mathrm{~g}$ a.i. ha ${ }^{-1}$ (PE) $f b 2,4-\mathrm{D}$ amine $1000 \mathrm{~g}$ a.i. ha ${ }^{-1}(\mathrm{PoE})$ was significantly superior in reducing weed density and dry weight of weeds. Weed free check, pendimethalin $1000 \mathrm{~g}$ a.i. ha ${ }^{-1}$ (PE) fb 2,4-D amine $1000 \mathrm{~g} \mathrm{a.i} \mathrm{ha-1.(PoE),}$ pendimethalin $1000 \mathrm{~g}$ a.i. ha ${ }^{-1}(\mathrm{PE}) \mathrm{fb}$ tembotrione $120 \mathrm{~g}$ a.i. ha $\mathrm{h}^{-1}$ (30 DAS) and atrazine $1000 \mathrm{~g} \mathrm{a.i.} \mathrm{ha}^{-1}$ (PE) $f b$ 2,4-D amine $1000 \mathrm{~g}$ a.i. ha ${ }^{-1}$ (PoE) registered 6335, 6099, 5549 and $5230 \mathrm{~kg} \mathrm{ha}^{-1}$ kernel yield respectively as against the seed yield of weedy check, $2737 \mathrm{~kg} \mathrm{ha}^{-1}$.
\end{abstract}

Keywords: Maize, weed management, new chemicals, pre-emergence and post-emergence

\section{Introduction}

Maize (Zea mays L.) or Indian corn is an essential crop which highly contributes to the world agriculture and more importantly to the world's food basket. In India, maize covers $9.28 \mathrm{~m}$ ha area with the productivity of 2.82 $\mathrm{MT} \mathrm{ha}^{-1}$ during 2018-19 (Ministry of Agriculture, India). Maize occupies importance as a human food (25\%), poultry supplement (49\%), animal supplement (12\%), and industrial products such as starch (12\%). Maize also contains maximum genetic yield potential, therefore maize is known as "Queen of cereals" (India Maize Summit, 2018). Weeds usually reduces crop yield up to $31.5 \%$ (22.7\% in rabi and in kharif $36.5 \%$ ). But as farmers adopt some kind of weeding on their crop field, a conservative estimate of $10 \%$ loss in crop yields may be taken as more realistic, hand weeding is most effective if done in time, though it is costly and time consuming. Apart from this, labourers are not available for weeding owing to other agricultural operation going on simultaneously. Additionally, manual method of weed control cannot be put into practice until weeds have achieved certain heights. In kharif maize, problem of severe weed infestation level combined with various species of weeds. Almost every type of weeds namely grassy, broad leaved and sedges infest the maize fields. As they compete for moisture, nutrients, space, light, shelter for many diseases and pest ultimately disturbs the growth of the plants, reduce the yield and also deteriorates the quality of crop. Sharma et al., (2000) ${ }^{[5]}$ reported that the reduction (33-50\%) in grain yield due to weed infestation. The higher loss in crop yield due to weed competition and it is evaluated that during the $1^{\text {st }} 3-6$ weeks (Shad et al., 1997) ${ }^{[4]}$. Therefore, there is a need for some alternate herbicides or sequential application of herbicide, which gives broad spectrum and season long control of weeds during the critical period in kharif maize, without affecting the crop growth and crop yield.

\section{Materials and methods}

A field experiment was conducted during kharif 2018-19 College of Agriculture, Latur to find out the effective herbicides for control of weeds in maize (Zea mays L.). The experiment comprises of ten treatment combinations and three replications under randomized block design with the net plot area $\left(16.56 \mathrm{~m}^{2}\right)$. The soil of experimental site was medium and black in color with good drainage and low in available nitrogen $\left(125.3 \mathrm{~kg} \mathrm{ha}^{-1}\right)$, medium in available phosphorous (18.20 kg ha-1) and very high in available potassium (498.58 kg ha $\mathrm{kg}^{-1}$. The soil was moderately alkaline in reaction having $\mathrm{pH}$ (7.7). Maize 'Mahabeej-Uday' was sown on 02 
August 2019 at $60 \mathrm{~cm}$ row to row and $30 \mathrm{~cm}$ plant to plant spacing using $15 \mathrm{~kg} \mathrm{ha}^{-1}$ seed rate and was harvested on 01 December 2019. Recommended dose of fertilizers $(150 \mathrm{~kg} \mathrm{~N}$ $+75 \mathrm{~kg} \mathrm{P}+75 \mathrm{~kg}, \mathrm{~kg} \mathrm{ha}^{-1}$ ) was applied. The doses of herbicides were computed as per treatments. The stock solution of desired concentration was prepared as per treatment for each plot and the herbicides were applied with knap sack sprayer. The pre-emergence herbicides were applied 24 hours after sowing and in case of post-emergence herbicides they were applied as per the treatments. Treatments were as follows, $\mathrm{T}_{1^{-}}$Weedy check, $\mathrm{T}_{2^{-}}$Weed free check, $\mathrm{T}_{3^{-}}$ Atrazine $1000 \mathrm{~g}$ a.i.ha ${ }^{-1}$ (PE) $\mathrm{fb}$ Halosulfuron-methyl $90 \mathrm{~g}$ a.i. $\mathrm{ha}^{-1}(\mathrm{PoE}), \mathrm{T}_{4}-$ Atrazine $1000 \mathrm{~g}$ a.i. ha ${ }^{-1}$ (PE) fb 2,4-D amine $1000 \mathrm{~g}$ a.i. ha ${ }^{-1}(\mathrm{PoE}), \mathrm{T}_{5}$ - Pendimethalin $1000 \mathrm{~g}$ a.i. ha ${ }^{-1}(\mathrm{PE})$ $f b$ halosulfuron-methyl $90 \mathrm{~g}$ a.i. ha ${ }^{-1}(\mathrm{PoE}), \mathrm{T}_{6}$ - Pendimethalin $1000 \mathrm{~g}$ a.i. ha ${ }^{-1}$ (PE) $f b$ tembotrione $120 \mathrm{~g}$ a.i. ha ${ }^{-1}$ (30 DAS), $\mathrm{T}_{7}$ - Pendimethalin $1000 \mathrm{~g}$ a.i. ha ${ }^{-1}$ (PE) fb 2,4-D amine $1000 \mathrm{~g}$ a.i. ha ${ }^{-1}(\mathrm{PoE}), \mathrm{T}_{8}-$ Halosulfuron-methyl $90 \mathrm{~g}$ a.i. ha ${ }^{-1}(\mathrm{PoE})$, $\mathrm{T}_{9-}$ Tembotrione $120 \mathrm{~g}$ a.i. $\mathrm{ha}^{-1}(\mathrm{PoE}), \mathrm{T}_{10^{-}}$2,4-D amine 1000 $\mathrm{g}$ a.i. ha ${ }^{-1}$ (PoE) (fb-Followed by). The observations on weed population was recorded at 30, 45 and 60 DAS and weed dry weight were recorded at harvest and data was analyzed using square root transformation $(X+0.5)$. The yield recorded at net plot basis converted to hectare and expressed in $\mathrm{kg} \mathrm{ha}^{-1}$. The data was statistically analyzed by adopting Fishers methods of analysis of variance as outlined by Gomez and Gomez (1984) ${ }^{[3]}$.

\section{Results and discussion \\ Weed dynamics}

The experimental field was infested with Commelina benghalensis L., Acalypha indica L., Amaranthus viridis, Euphorbia spp., Parthenium hysterophorun, Corchorus fascicularis L., Abutilon hirtum (Lam.), Boerhavia coccinea, Phyllanthus niruri, Argemone Mexicana L., Euphorbia geniculata Orteg., Achyranthus aspera L., Cardiospermum helicacabum L., Xanthium strumarium L., Celosia argentea L. under broad leaved weeds followed by grasses Sorghum halepense L., Cynodon dactylon L. Pers., Eriochloa spp. and sedges Cyperus rotundus L. At 30 DAS, significant reduction in total weed density was recorded under herbicidal treatment Pendimethalin $1000 \mathrm{~g}$ a.i.ha ${ }^{-1}$ (PE) $f b$ 2,4-D amine $1000 \mathrm{~g}$ a.i.ha ${ }^{-1}(\mathrm{PoE})$, which was at par with Atrazine $1000 \mathrm{~g}^{\text {a.i.ha }}{ }^{-1}$
(PE) $f b$ Halosulfuron-methyl $90 \mathrm{~g}$ a.i.ha ${ }^{-1}$ (PoE), Atrazine $1000 \mathrm{~g}$ a.i.ha ${ }^{-1}$ (PE) fb 2,4-D amine $1000 \mathrm{~g}$ a.i.ha ${ }^{-1}$ (PoE) and Pendimethalin $1000 \mathrm{~g}$ a.i. ha ${ }^{-1}$ (PE) $f b$ tembotrione $120 \mathrm{~g}$ a.i.ha ${ }^{-1}$ (30 DAS) but significantly superior over other herbicidal treatments. At 45 DAS, minimum total weed density was observed in Pendimethalin $1000 \mathrm{~g}$ a.i.ha ${ }^{-1}$ (PE) $\mathrm{fb}$ 2,4-D amine $1000 \mathrm{~g}$ a.i.ha ${ }^{-1}(\mathrm{PoE})$ which was at par with Pendimethalin $1000 \mathrm{~g}$ a.i.ha ${ }^{-1}$ (PE) $f b$ tembotrione $120 \mathrm{~g}$ a.i.ha ${ }^{-1}$ (30 DAS) and found significantly superior over other herbicidal treatments. At 60 DAS, significant reduction in total weed density was recorded under herbicidal treatment Pendimethalin $1000 \mathrm{~g}$ a.i.ha ${ }^{-1}$ (PE) fb 2,4-D amine $1000 \mathrm{~g}$ a.i.ha ${ }^{-1}(\mathrm{PoE})$, which was at par with Atrazine $1000 \mathrm{~g}$ a.i.ha ${ }^{-1}$ (PE) $f b$ 2,4-D amine $1000 \mathrm{~g}$ a.i.ha ${ }^{-1}$ (PoE) and Pendimethalin $1000 \mathrm{~g}$ a.i.ha ${ }^{-1}$ (PE) $f b$ tembotrione $120 \mathrm{~g}$ a.i.ha ${ }^{-1}$ (30 DAS) and found significantly superior over rest of the herbicidal treatments. Although, Weed free check showed lowest total weed density. Whereas highest total weed density was observed in Weedy check. Total dry weight of weeds was significantly reduced under Pendimethalin $1000 \mathrm{~g}$ a.i.ha ${ }^{-1}$ (PE) $f b$ 2,4-D amine $1000 \mathrm{~g}$ a.i.ha ${ }^{-1}$ (PoE) followed by Pendimethalin $1000 \mathrm{~g}$ a.i.ha ${ }^{-1}$ (PE) $f b$ tembotrione $120 \mathrm{~g}$ a.i.ha ${ }^{-1}$ (30 DAS) as compared to other herbicidal treatments. It might be due to two fold action of this combination and sequential application that affected both grasses as well as broad leaf weeds. The greater effectiveness of Pendimethalin $1000 \mathrm{~g}$ a.i.ha ${ }^{-1}$ (PE) $f b$ 2,4-D amine $1000 \mathrm{~g}$ a.i.ha ${ }^{-1}$ (PoE) has also been reported by Biswas et al. (2018) ${ }^{[2]}$ and Barua S. (2019) ${ }^{[1]}$, who observed least total weeds dry weight under these combination. Maximum weed index was observed in Weedy check, followed by Halosulfuron-methyl $90 \mathrm{~g}$ a.i.ha ${ }^{-1}$ (PoE), Tembotrione $120 \mathrm{~g}$ a.i.ha ${ }^{-1}$ (PoE) and Atrazine $1000 \mathrm{~g}$ a.i.ha ${ }^{-1}$ (PE) $f b$ Halosulfuron-methyl $90 \mathrm{~g}$ a.i.ha ${ }^{-1}$ (PoE). Whereas, minimum weed index was found in Weed free check followed by Pendimethalin $1000 \mathrm{~g}$ a.i.ha ${ }^{-1}$ (PE) $f b$ 2,4$\mathrm{D}$ amine $1000 \mathrm{~g}$ a.i.ha ${ }^{-1}$ (PoE), Pendimethalin $1000 \mathrm{~g}$ a.i.ha ${ }^{-1}$ (PE) $\mathrm{fb}$ tembotrione $120 \mathrm{~g}$ a.i.ha ${ }^{-1}$ (30 DAS), Atrazine $1000 \mathrm{~g}$ a.i.ha ${ }^{-1}$ (PE) $f b$ 2,4-D amine $1000 \mathrm{~g}$ a.i.ha ${ }^{-1}$ (PoE). Higher weed control efficiency and herbicide efficiency index among herbicidal treatments was noticed in Pendimethalin $1000 \mathrm{~g}$ a.i.ha ${ }^{-1}$ (PE) $f b$ 2,4-D amine $1000 \mathrm{~g}$ a.i.ha ${ }^{-1}$ (PoE) followed by Pendimethalin $1000 \mathrm{~g}$ a.i.ha ${ }^{-1}$ (PE) $f b$ tembotrione $120 \mathrm{~g}$ a.i.ha ${ }^{-1}$ (30 DAS) (Table 1).

Table 1: Effect of weed control treatments on density and dry weight of weeds, weed control efficiency (\%), weed index (\%), herbicide efficiency index $(\%)$, kernel yield $\left(\mathrm{kg} \mathrm{ha}^{-1}\right)$ and stover yield $\left(\mathrm{kg} \mathrm{ha}^{-1}\right)$

\begin{tabular}{|c|c|c|c|c|c|c|c|}
\hline \multirow[b]{2}{*}{ Treatment } & \multicolumn{3}{|c|}{ Weed density $\left(1 \mathrm{~m}^{2}\right)$} & \multirow{2}{*}{$\begin{array}{c}\text { Weed dry } \\
\text { weight (g/net } \\
\text { plot*) }^{*}\end{array}$} & \multirow{2}{*}{\begin{tabular}{|c|}
$\begin{array}{c}\text { Weed } \\
\text { control } \\
\text { efficiency }\end{array}$ \\
$(\%)$
\end{tabular}} & \multirow{2}{*}{$\begin{array}{c}\text { Weed } \\
\text { index } \\
(\%)\end{array}$} & \multirow{2}{*}{$\begin{array}{c}\text { Herbicide } \\
\text { efficiency } \\
\text { index } \\
(\%)\end{array}$} \\
\hline & 30 DAS & 45 DAS & 60 DAS & & & & \\
\hline Weedy check & $\begin{array}{l}6.19 * * \\
(37.83)\end{array}$ & $6.57(42.67)$ & $6.81(45.94)$ & $96.17(9250.06)$ & 0.00 & 131.42 & 0.00 \\
\hline Weed free check & $2.81(7.44)$ & $1.87(3.00)$ & $1.52(1.84)$ & $23.51(552.63)$ & 94.03 & 0.00 & 22.00 \\
\hline Atrazine $1000 \mathrm{~g}$ a.i.ha ${ }^{-1}$ (PE) fb Halosulfuron-methyl $90 \mathrm{~g}$ a.i.ha ${ }^{-1}(\mathrm{PoE})$ & $2.67(6.66)$ & $3.38(10.98)$ & $3.06(8.88)$ & $40.02(1601.36)$ & 82.69 & 50.91 & 3.08 \\
\hline Atrazine $1000 \mathrm{~g}$ a.i.ha ${ }^{-1}$ (PE) $f b$ 2,4-D amine $1000 \mathrm{~g}$ a.i.ha ${ }^{-1}(\mathrm{PoE})$ & $2.67(6.66)$ & $2.68(6.70)$ & $1.96(3.35)$ & $33.97(1153.46)$ & 87.53 & 21.12 & 7.30 \\
\hline Pendimethalin $1000 \mathrm{~g}$ a.i.ha ${ }^{-1}(\mathrm{PE}) f b$ halosulfuron-methyl $90 \mathrm{~g}$ a.i.ha ${ }^{-1}(\mathrm{PoE})$ & $3.05(8.83)$ & $3.1(9.11)$ & $2.75(7.11)$ & $35.37(1250.96)$ & 86.48 & 39.72 & 4.85 \\
\hline Pendimethalin $1000 \mathrm{~g}$ a.i.ha ${ }^{-1}$ (PE) $f b$ tembotrione $120 \mathrm{~g}$ a.i.ha ${ }^{-1}$ (30 DAS) & $2.49(5.72)$ & $2.05(3.71)$ & $1.75(2.57)$ & $30.95(957.73)$ & 89.65 & 14.15 & 9.92 \\
\hline Pendimethalin $1000 \mathrm{~g}$ a.i.ha $^{-1}$ (PE) $f b$ 2,4-D amine $1000 \mathrm{~g}$ a.i.ha ${ }^{-1}(\mathrm{PoE})$ & $2.35(5.04)$ & $1.91(3.16)$ & $1.74(2.56)$ & $27.32(745.9)$ & 91.94 & 3.86 & 15.23 \\
\hline Halosulfuron-methyl $90 \mathrm{~g}$ a.i.ha ${ }^{-1}(\mathrm{PoE})$ & $5.24(27.00)$ & $3.73(13.44)$ & $3.93(15.00)$ & $61.82(3821.43)$ & 58.69 & 79.85 & 0.69 \\
\hline Tembotrione $120 \mathrm{~g}$ a.i.ha ${ }^{-1}(\mathrm{PoE})$ & $5.29(27.58)$ & $2.96(8.27)$ & $2.90(7.93)$ & $60.5(3660.9)$ & 60.42 & 56.42 & 1.21 \\
\hline 2,4-D amine $1000 \mathrm{~g}^{2}$ a.i.ha ${ }^{-1}(\mathrm{PoE})$ & $5.22(26.83)$ & $2.91(8.00)$ & $2.30(4.79)$ & $34.07(1160.36)$ & 87.46 & 30.37 & 6.18 \\
\hline $\mathrm{SE} \pm$ & \begin{tabular}{|l|}
0.72 \\
\end{tabular} & 0.48 & 0.58 & 98.13 & - & \begin{tabular}{|l|l|}
- \\
\end{tabular} & - \\
\hline CD 5\% & 2.14 & 1.43 & 1.74 & 291.52 & - & - & - \\
\hline
\end{tabular}

$* 16.56 \mathrm{~m}^{2}, * *$ data in parenthesis (original value) was subjected to $\sqrt{\mathrm{X}}+0.5$ transformation.

\section{Effect on yield}

Data regarding cob yield (Table 2) showed that, statistically higher cob yield $\left(\mathrm{kg} \mathrm{ha}^{-1}\right)$ was observed in Weed free check i.e. $7940 \mathrm{~kg} \mathrm{ha}^{-1}$, which was at par with Pendimethalin $1000 \mathrm{~g}$ a.i.ha ${ }^{-1}$ (PE) fb 2,4-D amine $1000 \mathrm{~g}^{\text {a.i.ha }}{ }^{-1}$ (PoE) having cob yield $7888 \mathrm{~kg} \mathrm{ha}^{-1}$ and Pendimethalin $1000 \mathrm{~g}^{\text {a.i.ha }}{ }^{-1}$ (PE) $\mathrm{fb}$ 
tembotrione $120 \mathrm{~g}$ a.i.ha ${ }^{-1}$ (30 DAS) having cob yield $7257 \mathrm{~kg}$ $\mathrm{ha}^{-1}$, but significantly superior over other herbicidal treatments. Of all the treatments Weedy check showed the lower cob yield $\left(\mathrm{kg} \mathrm{ha}^{-1}\right)$ i.e. $4434 \mathrm{~kg} \mathrm{ha}^{-1}$ as compared to any other treatments. Among all the treatments, statistically higher kernel yield $\left(\mathrm{kg} \mathrm{ha}^{-1}\right)$ was observed in Weed free check i.e. $6335.23 \mathrm{~kg} \mathrm{ha}^{-1}$, which was at par with Pendimethalin $1000 \mathrm{~g}$ a.i.ha ${ }^{-1}$ (PE) $f b$ 2,4-D amine $1000 \mathrm{~g}$ a.i.ha ${ }^{-1}$ (PoE) having kernel yield $6099.73 \mathrm{~kg} \mathrm{ha}^{-1}$ and Pendimethalin $1000 \mathrm{~g}$ a.i. ha${ }^{1}$ (PE) $f b$ tembotrione $120 \mathrm{~g}$ a.i.ha ${ }^{-1}$ (30 DAS) having kernel yield $5549 \mathrm{~kg} \mathrm{ha}^{-1}$, but significantly superior over other herbicidal treatments. Of all the treatments Weedy check showed the lowest kernel yield $\left(\mathrm{kg} \mathrm{ha}^{-1}\right)$ i.e. $2737 \mathrm{~kg} \mathrm{ha}^{-1}$ as compared to any other treatments. Data indicated that the statistically higher stover yield $\left(\mathrm{kg} \mathrm{ha}^{-1}\right)$ was observed in Weed free check i.e. $11207.03 \mathrm{~kg} \mathrm{ha}^{-1}$, which was at par with Pendimethalin $1000 \mathrm{~g}$ a.i.ha ${ }^{-1}$ (PE) fb 2,4-D amine $1000 \mathrm{~g}$ a.i.ha ${ }^{-1}$ (PoE) having stover yield $9878.97 \mathrm{~kg} \mathrm{ha}^{-1}$ and Pendimethalin $1000 \mathrm{~g}$ a.i.ha ${ }^{-1}$ (PE) $f b$ tembotrione $120 \mathrm{~g}$ a.i.ha-1 (30 DAS) having stover yield $9091 \mathrm{~kg} \mathrm{ha}^{-1}$, but significantly superior over other herbicidal treatments. Of all the treatments Weedy check showed the low stover yield $(\mathrm{kg}$ $\mathrm{ha}^{-1}$ ) i.e. $6767 \mathrm{~kg} \mathrm{ha}^{-1}$ as compared to any other treatments. The yield (grain and stover) is the function of cumulative effect of yield attributes and the growth characters. The grain yield of maize positively influenced by cob length, cob girth, number of rows $\mathrm{cob}^{-1}$, number of kernels row $^{-1}$ and test weight. Yield attributes of maize were significantly influenced by adapting different weed management practices and higher value were noticed under treatments which had lower weed density and their dry weight and as a result of minimum weed competition. The reduction in weed competition in maize by the application of herbicides not only favored the crop plants with more availability of space, light, moisture and nutrients but also minimized weed interference, facilitating vigorous growth of crop plants. These results are found to be in close conformity with Biswas et al., (2018) ${ }^{[2]}$ and Barua S. (2019) ${ }^{[1]}$.

Table 2: Effect of weed control treatments on yield $\left(\mathrm{kg} \mathrm{ha}^{-1}\right)$

\begin{tabular}{|c|c|c|c|}
\hline Treatment & Cob Yield kg ha' & \begin{tabular}{|c|} 
Kernel Yield kg \\
ha $^{-1}$
\end{tabular} & $\begin{array}{c}\text { Stover Yield kg } \\
\text { ha }^{-1}\end{array}$ \\
\hline Weedy check & 4434 & \begin{tabular}{|l|l}
2737 \\
\end{tabular} & 6767 \\
\hline Weed free check & 7940 & 6335 & 11207 \\
\hline Atrazine $1000 \mathrm{~g}$ a.i.ha ${ }^{-1}(\mathrm{PE}) f b$ Halosulfuron-methyl $90 \mathrm{~g}$ a.i.ha ${ }^{-1}(\mathrm{PoE})$ & 5980 & 4198 & 7846 \\
\hline Atrazine $1000 \mathrm{~g}$ a.i.ha ${ }^{-1}(\mathrm{PE}) \mathrm{fb}$ 2,4-D amine $1000 \mathrm{~g}$ a.i.ha ${ }^{-1}(\mathrm{PoE})$ & 6933 & 5230 & 8216 \\
\hline Pendimethalin $1000 \mathrm{~g}$ a.i.ha ${ }^{-1}(\mathrm{PE}) \mathrm{fb}$ halosulfuron-methyl $90 \mathrm{~g}$ a.i.ha ${ }^{-1}(\mathrm{PoE})$ & 6343 & 4534 & 8077 \\
\hline Pendimethalin $1000 \mathrm{~g}$ a.i.ha ${ }^{-1}$ (PE) $f b$ tembotrione $120 \mathrm{~g}$ a.i.ha ${ }^{-1}$ (30 DAS) & 7257 & 5549 & 9091 \\
\hline Pendimethalin $1000 \mathrm{~g}_{\text {a.i.ha }}{ }^{-1}(\mathrm{PE}) f b$ 2,4-D amine $1000 \mathrm{~g}$ a.i.ha ${ }^{-1}(\mathrm{PoE})$ & 7888 & 6099 & 9878 \\
\hline Halosulfuron-methyl $90 \mathrm{~g}^{\mathrm{g}}$ a.i.ha ${ }^{-1}(\mathrm{PoE})$ & 4884 & 3522 & 7217 \\
\hline Tembotrione $120 \mathrm{~g}$ a.i.ha ${ }^{-1}(\mathrm{PoE})$ & 5764 & 4050 & 7302 \\
\hline 2,4-D amine $1000 \mathrm{~g}$ a.i.ha ${ }^{-1}(\mathrm{PoE})$ & 6434 & 4859 & 8117 \\
\hline $\mathrm{SE} \pm$ & 392 & 288 & 456 \\
\hline $\mathrm{CD} 5 \%$ & 1165 & 856 & 1355 \\
\hline
\end{tabular}

\section{Conclusion}

Based on the result it can be inferred that application of Pendimethalin $1000 \mathrm{gm}$ a.i. ha-1 (PE) fb 2,4-D amine $1000 \mathrm{gm}$ a.i. ha ${ }^{-1}$ (PoE) is proved to be most effective in controlling weeds, gave higher yield, although hand weeding is the best.

\section{References}

1. Barua S. Weed dynamics and productivity of maize (Zea mays L.) under pre and post emergence application of herbicide. M.Sc. (Agriculture) Thesis, I. G. K. V., Raipur 2019.

2. Biswas S, Debnath S, Saha A, Biswas B. Weed management in maize system in new alluvial zone of West Bengal, India. International Journal of Current Microbiology and Applied Science 2018;7(4):1344-1350.

3. Gomez KA, Gomez AA. Statistical procedure for agricultural research. John wiley and sons. New Delhi 1984, 680.

4. Shad RA, Chatna MQ, Nawaz H. Weed management studies in maize. Pakistan Journal of Agricultural Research 1997;14(1):44-50.

5. Sharma AR, Toor AS, Sur HS. Effect of interculture operation and scheduling of atrazine application on weed control and productivity of maize (Zea mays L.) in shiwalik foothills of Punjab. Indian Journal of Agricultural Science 2000;70:757-761. 